\title{
Use of Immunostaining for the diagnosis of Lymphovascular invasion in superficial Barrett's esophageal adenocarcinoma
}

Isao Hosono', Ryoji Miyahara 1* (D, Kazuhiro Furukawa', Kohei Funasaka', Tsunaki Sawada², Keiko Maeda², Takeshi Yamamura², Takuya Ishikawa', Eizaburo Ohno', Masanao Nakamura', Hiroki Kawashima', Takio Yokoi', Tetsuya Tsukamoto ${ }^{4}$, Yoshiki Hirooka ${ }^{2}$ and Mitsuhiro Fujishiro ${ }^{1}$

\begin{abstract}
Background: The prevalence of Barrett's esophageal adenocarcinoma (BEA) is increasing in Japan. Accurate assessment of lymphovascular invasion (LVI) after endoscopic resection or surgery is essential in evaluating treatment response. This study aimed to assess the usefulness of immunostaining in determining the extent of LVI in superficial BEA.

Methods: We retrospectively included 41 patients who underwent endoscopic resection or surgery between January 2007 and July 2018. In all cases, 3- $\mu$ m serial sections from paraffin-embedded resected specimens were used for hematoxylin and eosin (H-E) staining and immunostaining for D2-40 and CD31. Two specialized gastrointestinal pathologists (T.Y. and T.T.), blinded to clinical information, independently evaluated the extent of LVI from these specimens. The LVI-positivity rate was evaluated with respect to the depth of invasion, changes in the positivity rate on immunostaining, pathological characteristics of patients with LVI, lymph node metastasis or relapse, and course after treatment.

Results: H-E staining alone identified LVI in 7 patients (positivity rate: 17.1\%). Depths of invasion were categorized based on extension to the submucosa (SM) or deeper. On immunostaining for D2-40 and CD31, additional positivity was detected in 2 patients with SM1 and 1 SM3, respectively; LVI was detected in 10 patients (positivity rate: $24.4 \%$ ). LVI-positivity rates with invasion of the superficial muscularis mucosa (SMM)/lamina propria mucosa (LPM)/deep muscularis mucosa (DMM), SM 1, 2, and 3 were $0,75,28.6$, and 55.6\%, respectively.
\end{abstract}

Conclusions: Combined H-E staining and immunostaining is useful in diagnosing LVI in superficial BEA, particularly in endoscopically resected specimens.

Keywords: Barrett's esophageal adenocarcinoma, D2-40, CD31

\footnotetext{
* Correspondence: myhr@med.nagoya-u.ac.jp

'Department of Gastroenterology and Hepatology, Nagoya University

Graduate School of Medicine, 65 Tsurumai-cho, Showa-ku, Nagoya 466-8550,

Japan

Full list of author information is available at the end of the article
}

(c) The Author(s). 2020 Open Access This article is licensed under a Creative Commons Attribution 4.0 International License, which permits use, sharing, adaptation, distribution and reproduction in any medium or format, as long as you give appropriate credit to the original author(s) and the source, provide a link to the Creative Commons licence, and indicate if changes were made. The images or other third party material in this article are included in the article's Creative Commons licence, unless indicated otherwise in a credit line to the material. If material is not included in the article's Creative Commons licence and your intended use is not permitted by statutory regulation or exceeds the permitted use, you will need to obtain permission directly from the copyright holder. To view a copy of this licence, visit http://creativecommons.org/licenses/by/4.0/. The Creative Commons Public Domain Dedication waiver (http://creativecommons.org/publicdomain/zero/1.0/) applies to the data made available in this article, unless otherwise stated in a credit line to the data. 


\section{Background}

In Barrett's esophagus (BE), columnar epithelium replaces normal squamous epithelium in the distal esophagus owing to repeated esophageal inflammation, injury, and repair caused by regurgitation of gastric acid or bile $[1,2]$. The longitudinal extension of Barrett's mucosa covering the entire circumference of the esophagus for at least $3 \mathrm{~cm}$ and less than $3 \mathrm{~cm}$ is termed long-segment Barrett's esophagus and short-segment Barrett's esophagus, respectively [3]. Adenocarcinoma originating from $\mathrm{BE}$ is termed Barrett's esophageal adenocarcinoma (BEA). In Europe and the US, BEA accounts for approximately $60 \%$ of all esophageal cancer cases [4], and recent reports suggest a rapid rise in incidence, exceeding that of esophageal squamous cell carcinoma [5]. Meanwhile, BEA is less frequent in Japan, comprising only $4.7 \%$ of all esophageal cancer cases [6]. However, the incidence of gastroesophageal reflux disease (GERD) has recently increased in Japan owing to the introduction of a Western-style diet and a decrease in the incidence of Helicobacter pylori infection [7]. This change may increase the incidence of $\mathrm{BE}$, and consequently, BEA. Indeed, several studies have reported a slight increase in the incidence of BEA in Japan [8, 9]. The 5-year survival rate for advanced BEA without distant metastases is only $<20 \%$ [10]; thus, early diagnosis and treatment are essential.

Superficial BEA, in which the depth of cancer invasion is limited on submucosa, is primarily treated with surgery and endoscopic treatment as it has low risk for lymph node metastases. In Europe and the US, the primary treatment modality for BEA is endoscopic mucosal resection (EMR) combined with radiofrequency ablation (RFA) [11], while in Japan, the treatment involves endoscopic submucosal dissection (ESD) as en bloc resection. Additional treatment may be considered in cases extending to the deep muscularis mucosa (DMM) or deeper or with lymphovascular invasion (LVI). ESD, which facilitates en bloc resection, is more beneficial than EMR as it allows for fractional excision. ESD has been gradually introduced in Europe and the US [12].

However, given the rarity of BEA in Japan, no guidelines have been established for endoscopic resection of superficial BEA. Currently, endoscopic treatment is performed according to the guidelines for esophageal squamous cell carcinoma. With the increase in the number of indications for ESD, a multicenter cooperative study reported the possibility of expanding indications for ESD to superficial BEA. In the absence of both LVI and components of poorly differentiated carcinoma, lymph node metastases were not observed in BEA measuring $\leq 30$ $\mathrm{mm}$ in the maximum diameter and in those with $\leq 500$ $\mu \mathrm{m}$ infiltration to the SM. However, D2-40 or CD31/ CD34 immunostaining was not performed to examine the presence of LVI. Furthermore, no central pathological diagnosis was obtained [13]. To date, no study has investigated the extent of LVI using immunostaining in superficial BEA treated by endoscopic resection or surgery. Therefore, we aimed to evaluate the use of immunostaining in identifying LVI in patients with superficial BEA.

\section{Methods \\ Patients}

This retrospective study evaluated 41 patients with superficial BEA who underwent endoscopic resection or surgery between January 2007 and July 2018 at the Nagoya University Hospital. Those treated at other hospitals and who received preoperative chemotherapy were excluded. Data on clinical information, endoscopic findings, treatments, histopathological findings, and course after treatment were collected from the electronic charts.

\section{Diagnoses}

Pathological diagnoses were made according to the Japanese Classification of Esophageal Cancer 11th edition, published by the Japan Esophageal Society [3]. New muscularis mucosa can sometimes be found just below the columnar epithelium. In the Japanese classification of esophageal cancer, the primary muscularis mucosa is referred to as the deep muscularis mucosa (DMM), and the new muscularis mucosa is referred to as the superficial muscularis mucosa (SMM). Takubo et al. reported that the duplicated muscularis mucosa was found $71.6 \%$ of Barrett's esophageal adenocarcinoma specimens resected endoscopically in German patients [14]. Japan Esophageal Society classified the depth of tumor invasion into 6 groups as follows:

SMM; Carcinoma in situ or tumor has invaded the superficial muscularis mucosa.

LPM; Tumor has invaded the lamina propria mucosa.

DMM; Tumor has invaded the deep muscularis mucosa.

SM1; SM2; and SM3 involving $\leq 1 / 3$ of the superficial, middle, and deep layers of the resected specimen, respectively (Fig. 1). Among the endoscopically resected specimens, those with an SM infiltration of $\leq$ and $>$ $200 \mu \mathrm{m}$ were regarded as SM1 and 2, respectively. Furthermore, SM infiltration cases were sub-divided into two groups based on a depth of SM infiltration of < and $\geq 500 \mu \mathrm{m}$, each of which were evaluated.

\section{Immunostaining and $\mathrm{LVI}$ assessment}

To evaluate the presence of LVI, 3- $\mu \mathrm{m}$ serial sections were prepared from paraffin-embedded blocks of resected specimens. We used Podoplanin (D2-40) [15, 16] and CD31 [17] for immunostaining, which specifically stained the lymphatic and vascular endothelial cells, respectively. Of the serial sections, section 1 was stained 
(a)

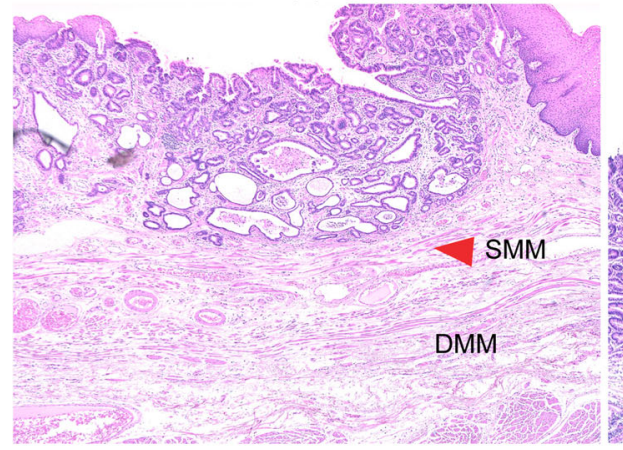

(c) (b)

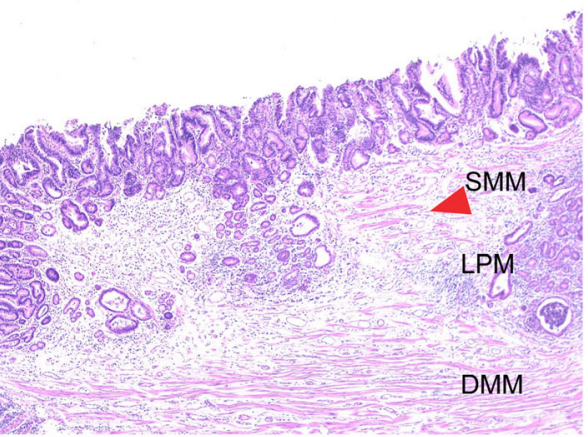

(d)

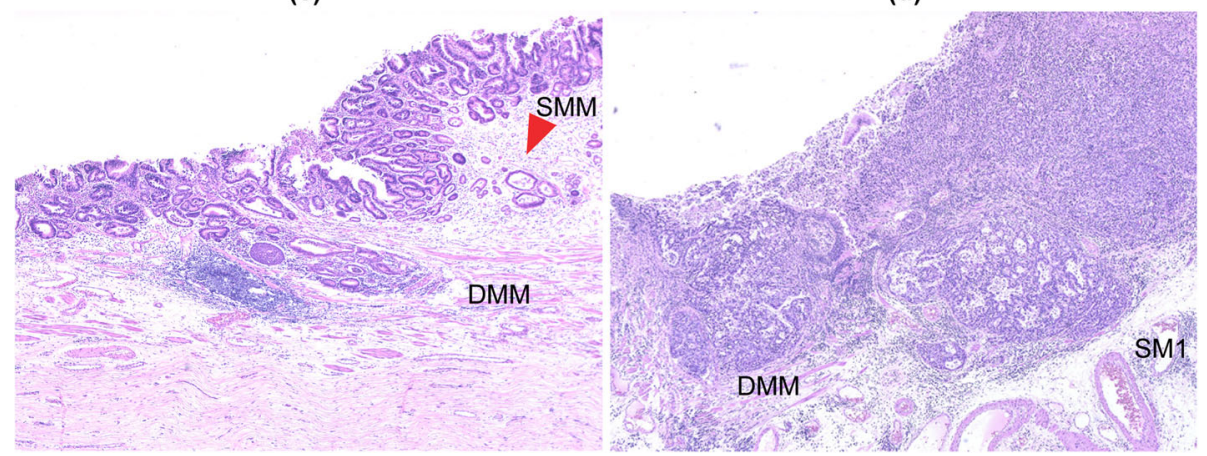

Fig. 1 a Tumor invasion to SMM. b Tumor invasion to LPM. c Tumor invasion to DMM. d Tumor invasion to SM (SM1). The red arrowhead indicates SMM. Abbreviations: superficial muscularis mucosa (SMM), lamina propria mucosa (LPM), deep mucularis mucosa (DMM),

submucosa (SM)

with D2-40, section 2 with H-E, and section 3 with CD31. Immunostaining was performed by using an automated immunostainer and iView ${ }^{\mathrm{mm}}$ DAB (3,3'-diaminobenzidine) Detection Kit (Ventana Medical Systems, Inc., Tucson, AZ, USA) with labeled streptavidin biotinylated antibody methods. Antigen retrieval was performed by heatinduced epitope retrieval methods using a citrate buffer (pH 8.5) and a steamer at $100^{\circ} \mathrm{C}$ for $60 \mathrm{~min}$. The sections were immunostained with an antihuman D2-40 monoclonal antibody (clone D2-40, Dako), a mouse monoclonal antibody (JC70, Roche Tissue Diagnostics) for CD31. Counterstaining was performed using hematoxylin.

LVI was microscopically assessed using the H-E- and immunostained specimens by two specialized gastrointestinal pathologists (T.Y. and T.T.) independently who were blinded to the clinical information. The evaluation of $\mathrm{H}-\mathrm{E}$ and immunostained specimens were performed independently at different times (rather than simultaneously). LVI was defined as endothelial cells recognizable on D2-40and CD31-positive cells and the presence of tumor cells in a space surrounded by these cells (Fig. 2).

The LVI-positivity rate was evaluated for the depth of invasion, changes in positivity rate on immunostaining, pathological characteristics of patients with LVI, lymph node metastasis or relapse, and treatment outcomes (overall, disease-specific, and relapse-free survival rates).

\section{Statistical analyses}

Continuous and categorical variables were presented as median (region) and number (percentage), respectively. Clinical parameters were compared using the MannWhitney U test and Fisher's exact test for continuous and categorical variables, respectively. The log-rank test was used to investigate the survival rate. A $p$-value of 0.05 was regarded as significant. All statistical analyses were performed using the IBM SPSS Statistics software version 25 (IBM SPSS, Chicago, IL, U.S.A.) package.

\section{Results}

\section{Patient characteristics}

The median age of the 41 patients was 67 years; the patient characteristics are detailed in Table 1. Macroscopically, protruding tumors were detected in 31 patients, and the median maximum tumor diameter was $20 \mathrm{~mm}$. ESD and surgery were performed as initial treatments in 13 and 28 patients, respectively. The histological types in 21, 17, and 3 patients were well differentiated (tub1), moderately differentiated (tub2), and poorly differentiated (por), respectively. Clinicopathological characteristics did not differ significantly between patients with short-segment versus longsegment Barrett's esophagus. 
(a)

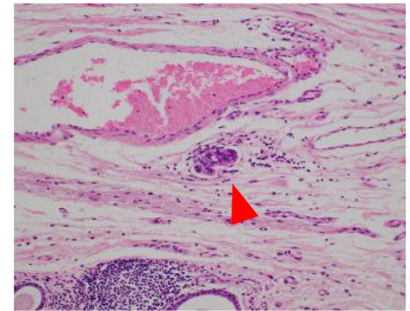

(d)

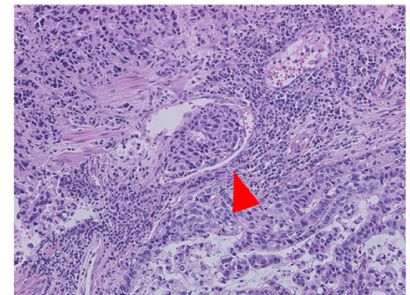

(b)

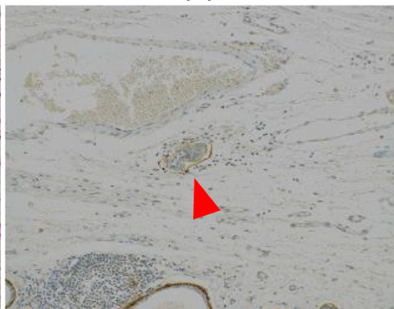

(e) (c)

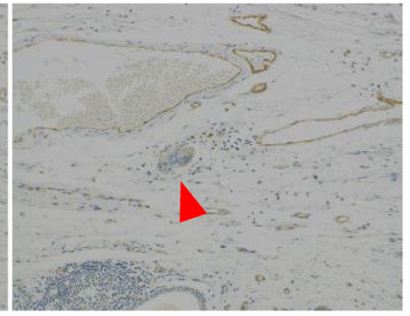

(f)

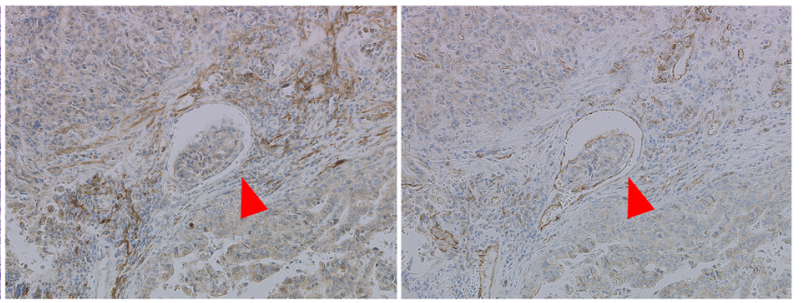

Fig. 2 a Microphotograph of lymphovascular invasion (LVI) as assessed using hematoxylin and eosin (H-E) staining. b Microphotograph of lymphatic vessel invasion as assessed using D2-40 staining (positive). c Microphotograph of blood vessel invasion as assessed using CD31 staining (negative). d Microphotograph of lymphovascular invasion (LVI) as assessed by hematoxylin and eosin (H-E) staining. e Microphotograph of lymphatic vessel invasion as assessed by D2-40 staining (negative). f Microphotograph of blood vessel invasion as assessed by CD31 staining (positive)

\section{Histopathological findings}

Table 2 shows the histological type and number of patients with LVI on $\mathrm{H}-\mathrm{E}$ staining and immunostaining for D2-40/CD31 according to the depth of invasion. Overall, 21 and 20 patients had pT1a and pT1b lesions, respectively, and 12 of the 21 patients with pT1a had
DMM lesions. The depth of SM infiltration in endoscopic resection exceeded $200 \mu \mathrm{m}$ in 3 patients, and the depths were 400, 800, and $1300 \mu \mathrm{m}$, respectively. Among them, 2 patients underwent additional surgery, which revealed no residual cancer or lymph node metastases. The remaining one patient opted not to have surgery

Table 1 Characteristics of patients treated by endoscopic submucosal dissection or surgery

\begin{tabular}{|c|c|c|c|c|}
\hline Characteristics & All patients $(n=41)$ & $\operatorname{SSBE}^{\mathrm{a}}(n=30)$ & $\operatorname{LSBE}^{\mathrm{b}}(n=11)$ & $\begin{array}{l}\text { SSBE VS. LSBE } \\
\text { P-value }\end{array}$ \\
\hline Age, median (range) & $67(39-81)$ & $66(39-88)$ & $68(44-79)$ & 0.757 \\
\hline \multicolumn{5}{|l|}{$\operatorname{Sex}(\%)$} \\
\hline Male & $32(78.0)$ & $23(76.7)$ & $9(81.8)$ & \multirow[t]{2}{*}{1.000} \\
\hline Female & $9(22.0)$ & $7(23.3)$ & $2(18.2)$ & \\
\hline Body mass index $\left(\mathrm{kg} / \mathrm{m}^{2}\right)$, median (range) & $23.0(16.7-32.6)$ & $23.1(16.7-32.6)$ & $23.0(16.7-32.6)$ & 0.596 \\
\hline Tumor size (mm), median (range) & $20(6-60)$ & $17.5(6-35)$ & $20(10-60)$ & 0.223 \\
\hline \multicolumn{5}{|l|}{ Macroscopic type (\%) } \\
\hline Protruding type & $31(75.6)$ & $25(83.4)$ & $6(54.5)$ & \multirow[t]{3}{*}{0.164} \\
\hline Flat type & $2(4.9)$ & $1(3.3)$ & $1(9.1)$ & \\
\hline Depressed type & $8(19.5)$ & $4(13.3)$ & $4(36.4)$ & \\
\hline \multicolumn{5}{|l|}{ Initial treatment (\%) } \\
\hline Endoscopic submucosal dissection (ESD) & $13(31.7)$ & $12(40.0)$ & $1(9.1)$ & \multirow[t]{2}{*}{0.127} \\
\hline Operation & $28(68.3)$ & $18(60.0)$ & $10(90.9)$ & \\
\hline \multicolumn{5}{|l|}{ Histological type (\%) } \\
\hline Well differentiated (tub1) & $21(51.2)$ & $18(60.0)$ & $4(36.4)$ & \multirow[t]{3}{*}{0.181} \\
\hline Moderately differentiated (tub2) & $17(41.5)$ & $11(36.7)$ & $5(45.4)$ & \\
\hline Poorly differentiated (por) & $3(7.3)$ & $1(3.3)$ & $2(18.2)$ & \\
\hline
\end{tabular}

${ }^{a} S S B E$ short-segment Barrett's esophagus ${ }^{\mathrm{b}}$ LSBE long-segment Barrett's esophagus 
Table 2 Histological Characteristics with Respect to Depth of Invasion, and Comparison of LVI-positivity Rates between H-E- and D240-/CD31-stained Specimens

\begin{tabular}{|c|c|c|c|c|c|c|c|c|c|c|c|c|}
\hline \multirow{2}{*}{\multicolumn{3}{|c|}{$\begin{array}{l}\text { Depth of invasion and } \\
\text { number }\end{array}$}} & \multicolumn{3}{|c|}{ Histological type } & \multicolumn{3}{|c|}{$\mathrm{H}$-E staining } & \multicolumn{3}{|c|}{ Immunostaining } & \multirow{2}{*}{$\begin{array}{l}P- \\
\text { value }\end{array}$} \\
\hline & & & tub1 & tub2 & por & $\mathrm{Ly}+$ & $V_{+}$ & LVI+ (\%) & D2-40 Ly+ & CD31 V+ & LVI+ (\%) & \\
\hline \multirow[t]{3}{*}{ T1a } & SMM & 7 & 6 & 1 & 0 & 0 & 0 & $0(0)$ & 0 & 0 & $0(0)$ & \\
\hline & LPM & 2 & 1 & 1 & 0 & 0 & 0 & $0(0)$ & 0 & 0 & $0(0)$ & \\
\hline & DMM & 12 & 10 & 2 & 0 & 0 & 0 & $0(0)$ & 0 & 0 & $0(0)$ & \\
\hline \multirow[t]{3}{*}{$\mathrm{T} 1 \mathrm{~b}$} & SM1 & 4 & 1 & 3 & 0 & 1 & 0 & $1(25)$ & 3 & 0 & $3(75)$ & \\
\hline & SM2 & 7 & 1 & 5 & 1 & 2 & 1 & $2(28.6)$ & 2 & 2 & $2(28.6)$ & \\
\hline & SM3 & 9 & 2 & 5 & 2 & 4 & 2 & $4(44.4)$ & 4 & 2 & $5(55.6)$ & \\
\hline \multicolumn{2}{|l|}{ Total } & 41 & & & & & & $7(17.1)$ & & & $10(24.4)$ & 0.587 \\
\hline
\end{tabular}

H-E Hematoxylin and eosin, SMM superficial muscularis mucosa, LPM lamina propria, DMM deep muscularis mucosa, SM submucosa

and instead was evaluated on follow-up; subsequently, she had no recurrence in 3-years following ESD. The incidences of histological subtypes with tub2 and por increased as invasion increased.

In 7 patients, LVI positivity was noted using H-Estained specimens alone (positivity rate: $17.1 \%$ ), and the depth of invasion was evaluated to be SM1 or deeper. LVI was found in 10 patients (positivity rate: $24.4 \%$ ) who were additionally diagnosed with LVI positivity on immunostaining for D2-40 and CD31. The concordance rate of LVI diagnosis between the two pathologists was 97.6\% (40/41) for H-E-stained specimens and 92.7\% (38/ 41) for immunostained specimens. The kappa coefficient for the two pathologists was 0.92 for $\mathrm{H}$-E-stained specimens and 0.82 for immunostained specimens, which indicated almost perfect agreement. The LVI-positivity rates in SMM, LPM, DMM, SM1, SM2, and SM3 lesions were $0,0,0,75,28.6$, and $55.6 \%$, respectively. Overall, between $\mathrm{H}-\mathrm{E}$ staining alone and immunostaining, LVI was consistently absent in $75.6 \%(31 / 41)$ cases. LVI was additionally detected on immunostaining in cases with SM1 (Fig. 3), in which the lymphatic endothelial cells were very thin near the tumor margin (site where LVI diagnosis is relatively easy), making recognition difficult, and in cases with SM3 (Fig. 4), in which the tumor volume was large, making the identification of LVI at the site of tumor infiltration impossible.
The patients in the SM group were sub-divided into two groups based on the depth of infiltration as follows: $<500 \mu \mathrm{m}$ and $\geq 500 \mu \mathrm{m}$. The former subgroup had $5 \mathrm{pa}-$ tients with SM1 lesions ( 4 and 1 underwent surgery and endoscopic resection with a depth of infiltration of $400 \mu \mathrm{m}$, respectively). The latter subgroup included 15 patients. LVI was present in $3(60 \%)$ of the patients with $<500 \mu \mathrm{m}$ submucosal infiltration and in $7(46.7 \%)$ of patients with $\geq 500 \mu \mathrm{m}$ submucosal infiltration. No specific pattern of distribution of LVI sites was observed.

\section{Lymph node metastasis and relapse}

Table 3 shows the pathological findings in 30 surgically treated patients with superficial BEA, who underwent surgical treatment, including 2 patients who underwent additional treatment after ESD. In total, $3 / 41$ (7.3\%) patients showed lymph node metastases. All three patients had protruding cancers derived from SSBE, invading at least up to SM2 (depth of infiltration: $>1000 \mu \mathrm{m}$ ). The tumor maximal diameters were $\geq 25 \mathrm{~mm}$, and they contained poorly differentiated components. LVI was identified in 2 of 3 patients.

\section{Overall survival rate and relapse-free survival}

The recurrence rate was slightly higher among patients with T1b disease. However, there were no significant differences in overall, disease-specific, and relapse-free

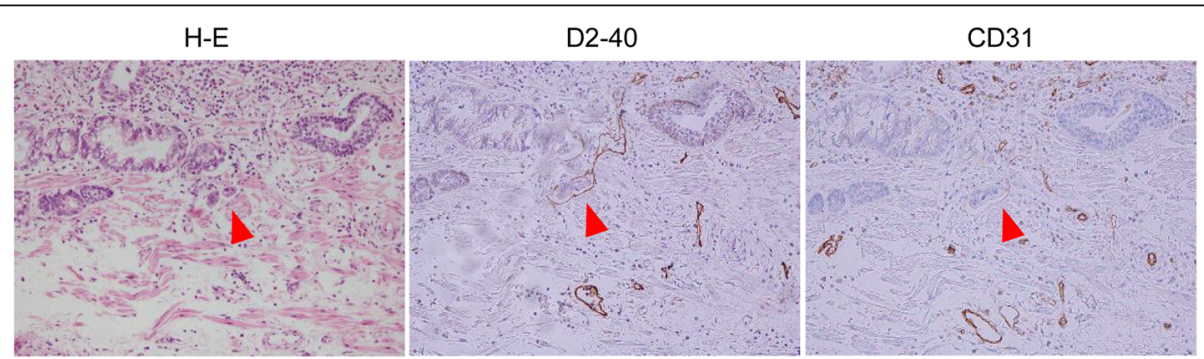

Fig. 3 A case where immunostaining was useful for evaluating the presence of lymphovascular invasion (patient with SM1, in whom the lymphatic endothelial cells were very thin, making recognition difficult. Lymphovascular invasion was detected on immunostaining for D2-40). Abbreviations: submucosa (SM) 


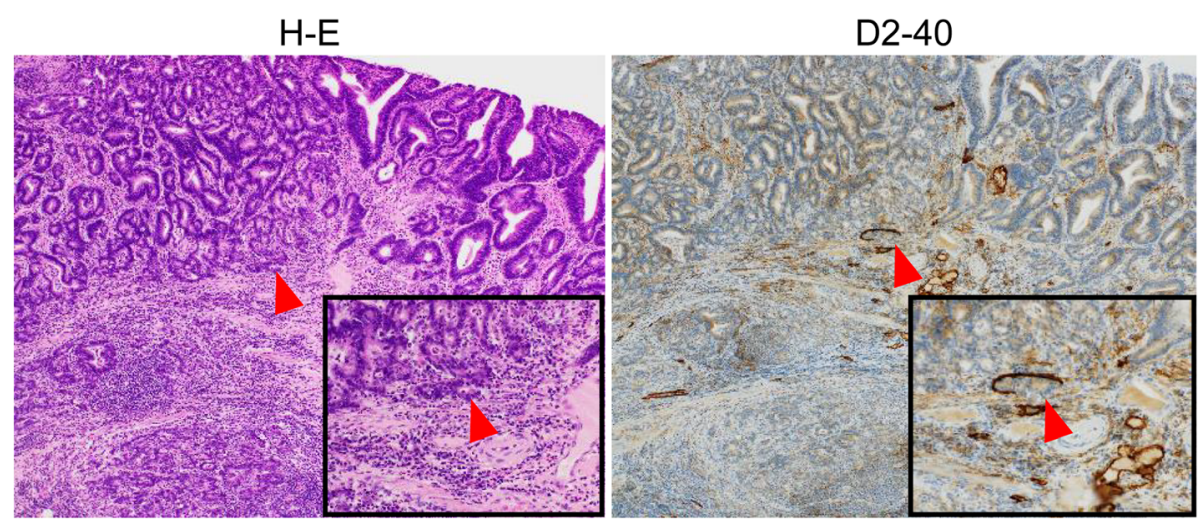

Fig. 4 A case where immunostaining was useful for evaluating the presence of lymphovascular invasion (patient with SM3, in whom the tumor volume was large, making the assessment of lymphovascular invasion at the site of tumor infiltration impossible. Lymphovascular invasion was detected on immunostaining with D2-40). Abbreviations: submucosa (SM)

survival between patients with T1a and T1b disease (Fig. 5). Relapse occurred in 3 patients with T1b disease, with a median follow-up of 46 months. In all 3 patients, LVI was present, the depth of invasion was evaluated to be at least SM2, poorly differentiated components were observed, and the tumor diameter was $\geq 20 \mathrm{~mm}$. Among them, 1 patient died of primary disease. The 3year disease-specific survival rate in those with T1a and T1b disease was 100 and 95.0\%, respectively.

\section{Discussion}

The results of this study show that combined H-E staining and immunostaining is useful in diagnosing LVI in superficial BEA, particularly in endoscopically resected specimens. LVI is directly related to lymph node/remote metastases in cancer patients [18-22]. Therefore, LVI may be useful in predicting the metastasis risk. In Japan, few studies have reported on the incidence of LVI positivity in BEA patients. Osumi et al. identified LVI in 18/55 lesions (32.7\%) with DMM [23]. Furthermore, Nishi et al. observed in lymphatic invasion in $10.3 \%$ of cases with DMM invasion. Further, also reported that the LVI-positivity rate increased with the depth of invasion [8].
In this study, LVI was present in patients with depths of invasion of at least SM1. The differences from previous reports were probably due to the number of patients and the use of immunostaining to identify LVI in all patients. Additional immunostaining increased the LVI-positivity rate by $7 \%$ than $\mathrm{H}-\mathrm{E}$ staining alone. In patients with SM1 lesions, this rate increased from 25 to $75 \%$. Although the number of SM1 patients was small $(n=4)$, the high positivity rate is noteworthy. LVI is usually assessed using $\mathrm{H}$ E-stained specimens. In patients in whom assessment is exceptionally difficult, the results may depend on the pathologist's subjective assessment [24, 25]. Particularly, it is difficult to evaluate fine lymphatic/venous invasion; difficult-to-identify lymphovascular endothelial cells; desmoplastic reaction of interstitial cells [26-28]; and artifacts related to tissue specimen preparation $[25,29,30]$. Here, LVI diagnosis was also difficult in some patients. Particularly, the difficulty in recognizing lymphatic/blood vessels may increase with a reduction in the grade of tumor differentiation. These factors limit LVI assessment using $\mathrm{H}$-E-stained specimens alone. Additional immunostaining may have increased the LVI-positivity rate among SM infiltrating lesions in this cohort.

Table 3 Pathological Findings in 30 Patients with Superficial Cancer who Underwent Surgery

\begin{tabular}{|c|c|c|c|c|c|c|c|}
\hline \multirow{2}{*}{$\begin{array}{l}\text { Depth of } \\
\text { invasion }\end{array}$} & \multirow{2}{*}{$\begin{array}{l}\text { Number of } \\
\text { patients }\end{array}$} & \multicolumn{3}{|c|}{ Histological type } & \multirow{2}{*}{$\begin{array}{l}\text { Lymphovascular invasion } \\
\mathrm{LVI+}\end{array}$} & \multirow{2}{*}{$\begin{array}{l}\text { Lymph node metastasis } \\
+\end{array}$} & \multirow{2}{*}{$\begin{array}{l}\text { Recurrence } \\
+\end{array}$} \\
\hline & & tub1 & tub2 & por & & & \\
\hline SMM & 3 & 3 & 0 & 0 & 0 & 0 & 0 \\
\hline LPM & 2 & 1 & 1 & 0 & 0 & 0 & 0 \\
\hline DMM & 6 & 5 & 1 & 0 & 0 & 0 & 0 \\
\hline SM1 & 4 & 1 & 3 & 0 & 3 & 0 & 0 \\
\hline SM2 & $6^{*}$ & 1 & 4 & 1 & 2 & 2 & 2 \\
\hline SM3 & 9 & 3 & 4 & 2 & 5 & 1 & 1 \\
\hline Total & 30 & 14 & 13 & 3 & 10 & 3 & 3 \\
\hline
\end{tabular}

*Including 2 patients who underwent additional surgery after ESD

SMM superficial muscularis mucosa, LPM lamina propria, DMM deep muscularis mucosa, SM submucosa 

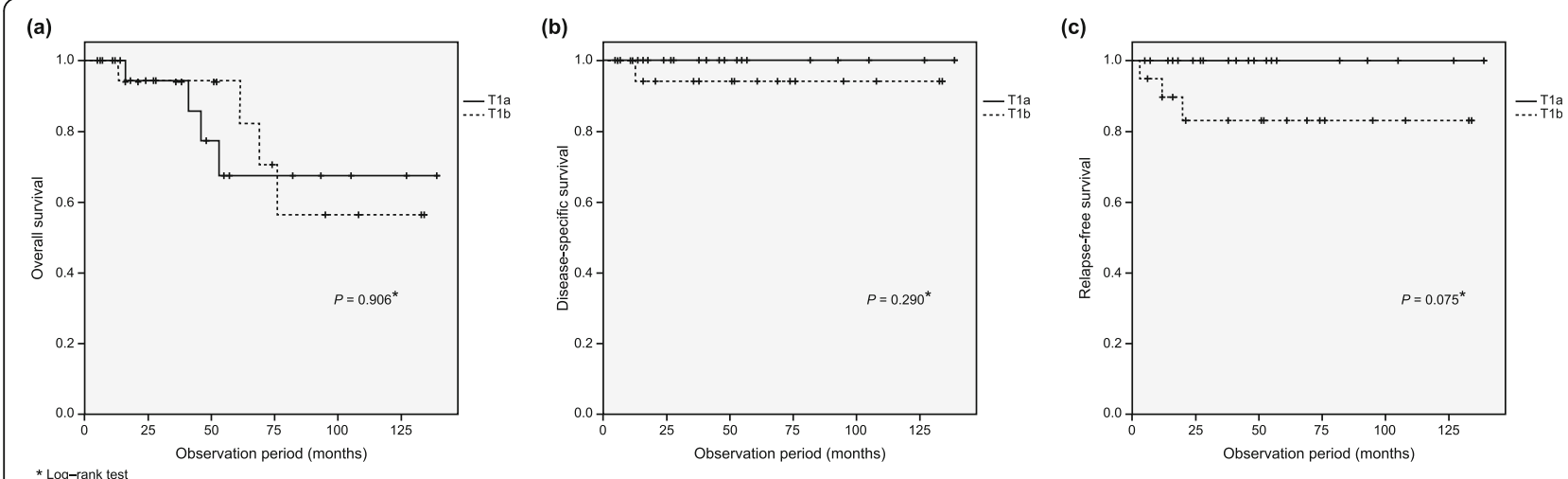

Fig. 5 Survival curves in patients with $T 1 a / T 1 b$ tumors. a Overall survival. b Disease-specific survival. c Relapse-free survival

Japanese guidelines recommend endoscopic treatment for early esophageal cancer and early gastric cancer. Conversely, no treatment guidelines for BEA have been developed owing to lack of data. In this study, LVI was absent in patients with infiltration up to the DMM. In SM1 lesions, no lymph node metastases were observed when the criteria proposed by Ishihara et al. were fulfilled [13]. This suggests that ESD may be increasingly employed in these cases. Notably, LVI, invasion to SM2 or deeper, presence of poorly differentiated components, and a maximum tumor diameter of $\geq 20 \mathrm{~mm}$ were common among patients with relapse. The patients with SM or superficial lesions had relatively favorable prognosis, and only few patients had relapse. Therefore, the risks of lymph node metastasis and relapse may be low in SM (infiltration: $<500 \mu \mathrm{m}$ ) lesions with a maximum diameter of $\leq 20 \mathrm{~mm}$, absence of LVI, and absence of poorly differentiated carcinoma components. This suggests that after ESD, follow-up is a feasible option in patients ineligible for surgery. However, LVI is detected on immunostaining in some patients with SM1 invasion. Therefore, pathological findings should be carefully evaluated with additional immunostaining.

The risk of lymph node metastasis must be adequately evaluated. Many studies reported that LVI, detected on immunostaining for D2-40, was an independent prognostic factor for lymph node metastasis [20, 21, 31, 32]. LVI diagnosis may predict subsequent lymph node metastasis. In this study, only few patients had lymph node metastasis or relapse, making detailed statistical analysis unreliable.

As a result of immunostaining, LVI was newly diagnosed in some patients and ruled out in some cases despite positivity on $\mathrm{H}$-E staining. Although we were unable to conclude statistically whether additional immunostaining significantly increased the LVI-positivity rate in comparison with $\mathrm{H}-\mathrm{E}$ staining alone, immunostaining may be useful in individual patients. The LVI-positivity rate was high among those with SM1 invasion; this should be considered while selecting patients for ESD. Furthermore, it is important to be able to identify the presence of LVI for predicting future relapse in patients with SM2 and SM3. In addition, positive findings on additional immunostaining in endoscopically resected specimens may facilitate decision-making for further treatment and prevent unnecessary surgery. However, immunostaining is cost and effort intensive and should be considered carefully in limited-resource settings.

The limitations of this study are the single-center retrospective design and small sample size. However, immunostaining for D2-40 and CD31 was performed in all patients with superficial BEA who underwent ESD or surgery, and the presence of LVI was examined. Furthermore, the proportion of surgically treated patients was relatively high; the number of evaluable cases with lymph node metastases was also large.

\section{Conclusion}

Immunostaining for D2-40 and CD31 is useful for identifying the presence of LVI in patients with superficial BEA. This is essential for evaluating the need for additional treatment, particularly in endoscopically resected specimens. Prospective multicenter studies on ESD and surgery as treatment options for superficial cancer, are needed.

\section{Abbreviations}

SMM: superficial muscularis mucosa; LPM: lamina propria mucosa; DMM: deep muscularis mucosa; SM: submucosa; BEA: Barrett's esophageal adenocarcinoma; BE: Barrett's esophagus; ESD: endoscopic submucosal dissection

\section{Acknowledgements}

None.

\section{Authors' contributions}

Study concept and design: IH and RM; Data acquisition: KaF, KoF, TS, KM, TYa, TI, EO, MN, and HK; Data analysis and interpretation: $\mathrm{H}, \mathrm{RM}$, and MF; Drafting of the manuscript: $\mathrm{H}$; Critical revision of the manuscript for important intellectual content: TYo and YH; Technical, or material support: TYo and TT. All authors approved the final version of the manuscript. 


\section{Funding}

None.

\section{Availability of data and materials}

The datasets used and/or analyzed during the current study are available from the corresponding author on reasonable request.

\section{Ethics approval and consent to participate}

The study protocol was approved by the ethics review board of the Nagoya University (Approval Number: 2017--0392), and the work performed in this study was in accordance with the principles of the Declaration of Helsinki. We got ""opt-out consent"" approved by "'Nagoya University Ethics Committee" ${ }^{\prime \prime \prime}$ as observational study under Japanese Code of Ethics.

\section{Consent for publication}

Not applicable.

\section{Competing interests}

Isao Hosono, Ryoji Miyahara, Kazuhiro Furukawa, Kohei Funasaka, Tsunaki Sawada, Keiko Maeda, Takeshi Yamamura, Takuya Ishikawa, Eizaburo Ohno, Masanao Nakamura, Hiroki Kawashima, Takio Yokoi, Tetsuya Tsukamoto, Yoshiki Hirooka and Mitsuhiro Fujishiro declare that they have no conflict of interest.

\section{Author details}

'Department of Gastroenterology and Hepatology, Nagoya University Graduate School of Medicine, 65 Tsurumai-cho, Showa-ku, Nagoya 466-8550, Japan. ${ }^{2}$ Department of Endoscopy, Nagoya University Hospital, Nagoya, Japan. ${ }^{3}$ Department of Pathology, Nagoya University Hospital, Nagoya, Japan. ${ }^{4}$ Department of Diagnostic Pathology, Fujita Health University School of Medicine, Toyoake, Aichi, Japan.

\section{Received: 24 January 2020 Accepted: 25 May 2020}

\section{Published online: 05 June 2020}

\section{References}

1. Hassall E. Barrett's esophagus: congenital or acquired? Am J Gastroenterol. 1993;88:819-24.

2. Eisen GM, Sandler RS, Murray S, et al. The relationship between gastroesophageal reflux disease and its complications with Barrett's esophagus. Am J Gastroenterol. 1997;92:27-31.

3. Japan Esophageal Society. Japanese classification of esophageal Cancer, 11th edition part I. Esophagus. 2017;14:1-36.

4. Rubenstein $\mathrm{JH}$, Shaheen NJ. Epidemiology, diagnosis, and management of esophageal adenocarcinoma. Gastroenterology. 2015;149:302-17 e1.

5. Everhart JE, Ruhl CE. Burden of digestive diseases in the United States part l: overall and upper gastrointestinal disease. Gastroenterology. 2009;136:376-86

6. Tachimori $Y$, Ozawa S, Numasaki H, Fujishiro M, Matsubara H, Oyama T, et al. Comprehensive registry of esophageal Cancer in Japan, 2009. Esophagus. 2016:13:110-37.

7. Fujiwara Y, Arakawa T. Epidemiology and clinical characteristics of GERD in the Japanese population. J Gastroenterol. 2009;44:518-34.

8. Nishi T, Makuuchi H, Ozawa S, Shimada H, Chino O. The present status and future of Barrett's esophageal adenocarcinoma in Japan. Digestion. 2019;99: 185-90.

9. Koizumi S, Motoyama S, lijima K. Is the incidence of esophageal adenocarcinoma increasing in Japan? Trends from the data of a hospitalbased registration system in Akita prefecture. Japan J Gastroenterol. 2018;53: 827-33.

10. Gillison EW, Powell J, McConkey CC, Spychal RT. Surgical workload and outcome after resection for carcinoma of the oesophagus and cardia. $\mathrm{Br} J$ Surg. 2002;89:344-8.

11. Shaheen NJ, Falk GW, lyer PG, Gerson LB. ACG clinical guideline: diagnosis and management of Barrett's esophagus. Am J Gastroenterol. 2016;111:30 50 quiz 51.

12. Höbel S, Dautel P, Baumbach R, Oldhafer KJ, Stang A, Feyerabend B, et al. Single center experience of endoscopic submucosal dissection (ESD) in early Barrett's adenocarcinoma. Surg Endosc. 2015;29:1591-7.

13. Ishihara R, Oyama T, Abe S, Takahashi H, Ono H, Fujisaki J, et al. Risk of metastasis in adenocarcinoma of the esophagus: a multicenter retrospective study in a Japanese population. J Gastroenterol. 2017;52:800-8.
14. Takubo K, Aida J, Naomoto Y, Sawabe M, Arai T, Shiraishi H, et al. Cardiac rather than intestinal-type background in endoscopic resection specimens of minute Barrett adenocarcinoma. Hum Pathol. 2009;40:65-74.

15. Kahn HJ, Bailey D, Marks A. Monoclonal antibody D2-40, a new marker of lymphatic endothelium, reacts with Kaposi's sarcoma and a subset of angiosarcomas. Mod Pathol. 2002;15:434-40.

16. Kaiserling E. Immunohistochemical identification of lymph vessels with D240 in diagnostic pathology. Pathologe. 2004;25:362-74.

17. Parums DV, Cordell JL, Micklem K, Heryet AR, Gatter KC, Mason DY, et al. JC70: a new monoclonal antibody that detects vascular endothelium associated antigen on routinely processed tissue sections. J Clin Pathol. 1990;43:752-7.

18. Estrella JS, Hofstetter WL, Correa AM, Estrella JS, Hofstetter WL, Correa AM, Swisher SG, Ajani JA, Lee JH, et al. Duplicated muscularis mucosae invasion has similar risk of lymph node metastasis and recurrence-free survival as intramucosal esophageal adenocarcinoma. Am J Surg Pathol. 2011;35:1045-53.

19. Raica M, Ribatti D. Targeting tumor lymphangiogenesis: an update. Curr Med Chem. 2010;17:698-708.

20. Tomita N, Matsumoto T, Hayashi T, Arakawa A, Sonoue H, Kajiyama Y, et al. Lymphatic invasion according to D2-40 immunostaining is a strong predictor of nodal metastasis in superficial squamous cell carcinoma of the esophagus: algorithm for risk of nodal metastasis based on lymphatic invasion. Pathol Int. 2008;58:282-7.

21. Weber SK, Sauerwald A, Pölcher M, Braun M, Debald M, Serce NB, et al. Detection of lymphovascular invasion by D2-40 (podoplanin) immunoexpression in endometrial cancer. Int J Gynecol Cancer. 2012;22: 1442-8.

22. Ukai R, Hashimoto K, Nakayama H, Iwamoto T. Lymphovascular invasion predicts poor prognosis in high-grade PT1 bladder cancer patients who underwent transurethral resection in one piece. Jpn J Clin Oncol. 2017;47:447-52.

23. Osumi H, Fujisaki J, Omae M, Shimizu T, Yoshio T, Ishiyama A, et al. Clinicopathological features of Siewert type II adenocarcinoma: comparison of gastric cardia adenocarcinoma and Barrett's esophageal adenocarcinoma following endoscopic submucosal dissection. Gastric Cancer. 2017;20:663-70.

24. Fan L, Mac MT, Frishberg DP, Fan X, Dhall D, Balzer BL, et al. Interobserver and intraobserver variability in evaluating vascular invasion in hepatocellular carcinoma. J Gastroenterol Hepatol. 2010;25:1556-61.

25. Harris El, Lewin DN, Wang HL, Lauwers GY, Srivastava A, Shyr Y, et al. Lymphovascular invasion in colorectal cancer: an interobserver variability study. Am J Surg Pathol. 2008;32:1816-21.

26. De Wever $\mathrm{O}$, Mareel $\mathrm{M}$. Role of tissue stroma in cancer cell invasion. J Pathol. 2003:200:429-47.

27. Ohtani H. Stromal reaction in cancer tissue: pathophysiologic significance of the expression of matrix-degrading enzymes in relation to matrix turnover and immune/inflammatory reactions. Pathol Int. 1998;48:1-9.

28. Hewitt RE, Powe DG, Carter GI, Turner DR. Desmoplasia and its relevance to colorectal tumour invasion. Int J Cancer. 1993;53:62-9.

29. Kojima M, Shimazaki H, Iwaya K, Kage M, Akiba J, Ohkura $Y$, et al. Pathological diagnostic criterion of blood and lymphatic vessel invasion in colorectal cancer: a framework for developing an objective pathological diagnostic system using the Delphi method, from the pathology working Group of the Japanese Society for Cancer of the Colon and Rectum. J Clin Pathol. 2013:66:551-8.

30. Okamoto $Y$, Fujimori T, Ohkura Y, Sugai T, Arai T, Watanabe G, et al. Histological assessment of intra- and inter-institutional reliabilities in detection of desmoplastic reaction in biopsy specimens of early colorectal carcinomas. Pathol Int. 2013:63:539-45.

31. Kozłowski M, Naumnik W, Nikliński J, Milewski R, Łapuć G, Laudański J. Lymphatic vessel invasion detected by the endothelial lymphatic marker D2-40 (podoplanin) is predictive of regional lymph node status and an independent prognostic factor in patients with resected esophageal cancer. Folia Histochem Cytobiol. 2011;49:90-7.

32. Wada H, Shiozawa M, Sugano N, Morinaga S, Rino Y, Masuda M, et al. Lymphatic invasion identified with D2-40 immunostaining as a risk factor of nodal metastasis in T1 colorectal cancer. Int J Clin Oncol. 2013 Dec;18(6):1025-31.

\section{Publisher's Note}

Springer Nature remains neutral with regard to jurisdictional claims in published maps and institutional affiliations. 